The following paper was published in the Journal of the Optical Society of America $A$ and is made available as an electronic reprint with the permission of OSA. The paper can also be found at the following URL on the OSA website:

http://www.opticsinfobase.org/abstract.cfm?URI=josaa-24-5-1438 


\title{
Adaptive optics retinal imaging reveals S-cone dystrophy in tritan color-vision deficiency
}

\author{
Rigmor C. Baraas \\ Department of Optometry and Visual Science, Buskerud University College, 3603 Kongsberg, Norway \\ Joseph Carroll and Karen L. Gunther \\ Department of Ophthalmology, The Medical College of Wisconsin, Milwaukee, Wisconsin 53226, USA
}

Mina Chung

Department of Ophthalmology, University of Rochester, Rochester, New York 14627, USA

David R. Williams

Center for Visual Science, University of Rochester, Rochester, New York 14627, USA

David H. Foster

School of Electrical and Electronic Engineering, University of Manchester, Manchester M60 1QD, UK

Maureen Neitz

Department of Ophthalmology, The Medical College of Wisconsin, Milwaukee, Wisconsin 53226, USA

Received September 1, 2006; revised December 6, 2006; accepted December 7, 2006; posted December 18, 2006 (Doc. ID 74659); published April 11, 2007

\begin{abstract}
Tritan color-vision deficiency is an autosomal dominant disorder associated with mutations in the shortwavelength-sensitive- (S-) cone-pigment gene. An unexplained feature of the disorder is that individuals with the same mutation manifest different degrees of deficiency. To date, it has not been possible to examine whether any loss of S-cone function is accompanied by physical disruption in the cone mosaic. Two related tritan subjects with the same novel mutation in their S-cone-opsin gene, but different degrees of deficiency, were examined. Adaptive optics was used to obtain high-resolution retinal images, which revealed distinctly different S-cone mosaics consistent with their discrepant phenotypes. In addition, a significant disruption in the regularity of the overall cone mosaic was observed in the subject completely lacking S-cone function. These results taken together with other recent findings from molecular genetics indicate that, with rare exceptions, tritan deficiency is progressive in nature. (C) 2007 Optical Society of America

OCIS codes: $330.1720,330.5310,100.0100,330.4300$.
\end{abstract}

\section{INTRODUCTION}

Tritan color-vision deficiency is an inherited autosomal dominant abnormality of short-wavelength-sensitive- (S-) cone function. 1,2 The disorder exhibits incomplete penetrance, meaning that individuals with the same underlying mutation manifest different degrees of color-vision impairment, even within a sibship. ${ }^{1,3-8}$ Mutations in the S-cone-opsin gene, which encodes the protein component of the S-cone photopigment, have been identified, and they give rise to four different single amino acid substitutions that have been found only in affected individuals and not in unaffected control subjects. ${ }^{9-11}$ Each substitution occurs at an amino acid position that lies in one of the transmembrane alpha helices and is predicted to interfere with folding, processing, or stability of the encoded opsin. For example, one of the mutations identified by Weitz et $a l .{ }^{10}$ corresponds to an amino acid position at which a substitution in the rod pigment rhodopsin causes autosomal dominant retinal degeneration. ${ }^{12,13}$

A fundamental property differentiating $\mathrm{S}$ cones from long-wavelength sensitive (L) and medium-wavelengthsensitive (M) cones is the potential for dominant negative interactions between normal and mutant opsins. This is because each $\mathrm{S}$ cone expresses both autosomal copies of the $\mathrm{S}$-opsin gene, whereas $\mathrm{L}$ and $\mathrm{M}$ cones each express only one opsin gene from the X chromosome. Rod photoreceptors also express a rhodopsin gene from two autosomes, and for rhodopsin mutations underlying autosomal dominant retinitis pigmentosa (adRP), dominant negative interactions lead to the death of the photoreceptors and ultimately to the degeneration of the retina. Tritan color-vision deficiencies have not been reported to cause progressive retinal degeneration, possibly because the proportion of $\mathrm{S}$ cones in the retina is much smaller 
than that of rods. ${ }^{10}$ It thus remains a clear possibility that $\mathrm{S}$ cones degenerate in autosomal dominant tritanassociated mutations of the $\mathrm{S}$ opsin.

Here, results are reported from experiments using adaptive optics to image the cones of tritan subjects whose molecular defect is known. These images have, for the first time, provided insight into the physical status of $\mathrm{S}$ cones in tritan color-vision deficiencies associated with S-opsin mutations. The results suggest that the colorvision deficiency is associated with a progressive S-cone dystrophy and accompanied by a disruption in the regularity of the cone mosaic.

\section{METHODS}

\section{A. Subjects}

Two tritan subjects, aged 57 and 34 years, took part in the experiments: one male (M04) and his daughter (M505). The father's tritan son (M503, aged $36 \mathrm{yr}$ ) was also included in the first part of the experiment on color-vision testing and genetics. A complete ophthalmic examination was carried out on the father and the daughter. The experiments were conducted in accordance with principles embodied in the Declaration of Helsinki (Code of Ethics of the World Medical Association) and were approved by the Research Ethics Committee of the University of Manchester (genetics and color-vision testing) and the institutional research board at the Medical College of Wisconsin (genetics) and the University of Rochester (adaptive optics imaging). Six normal trichromats [2 male, 4 female; mean age, $26 \mathrm{yr}$; standard deviation (SD), $3.7 \mathrm{yr}$ ] served as controls for the retinal-imaging experiments.

\section{B. Color-Vision Testing}

Subjects were classified with a variety of clinical colorvision tests: the Farnsworth-Munsell 100-Hue test (FM 100-Hue); Ishihara pseudoisochromatic plates (24-plate edition, 1964); and Rayleigh and Moreland anomaloscopy and luminance matching (Interzeag Color Vision meter 712, Schlieren, Switzerland). The father and daughter were also tested on the HRR pseudoisochromatic plates (4th edition, 2002); the Lanthony's desaturated D15; the Farnsworth D15; and the Medmont C100; the father was additionally tested on the Cambridge Color Test. ${ }^{14}$

\section{DNA Isolation and Genetic Analyses}

Whole blood was obtained from each subject, and genomic DNA was extracted using the PureGene kit (Gentra Systems, Minneapolis). The five exons that comprise the amino acid coding region of the S-opsin gene were amplified in the polymerase chain reaction (PCR) and were sequenced following previously described methods. ${ }^{9}$ Sequencing reactions were analyzed on an ABI Prism 3100xl genetic analyzer (Applied Biosystems, Foster City, California). Twenty-nine nontritan subjects served as a control population on the genetic analysis, and their S-opsin genes were also sequenced.

\section{Imaging}

High-resolution retinal imaging was carried out with the Rochester Second Generation Adaptive Optics System, which has been described in detail elsewhere. ${ }^{15}$ The subject's head was stabilized with a dental-impression bite bar. The subject's right or left eye was dilated, and accommodation was suspended with a combination of phenylephrine hydrochloride $(2.5 \%)$ and tropicamide (1\%). Measurements of the eye's monochromatic aberrations were taken over a 6.8-mm pupil with a Shack-Hartmann wavefront sensor and corrected with a 97-channel deformable mirror (Xinetics, Inc., Devins, Massachusetts) in a continuous closed-loop fashion until the root-mean-square wavefront error fell below $0.1 \mu \mathrm{m}$ or $800 \mathrm{~ms}$ had elapsed, whichever happened first. For further details on wavefront measurements and compensation, see Refs. 15-17. Once a wavefront correction was obtained, a retinal image was acquired by illuminating the retina with a 1-deg-diameter, 4-ms flash at $550 \mathrm{~nm}$ with $25-\mathrm{nm}$ bandwidth (full width at half-maximum) from a krypton-arc flash lamp. The short duration of the flash helped to minimize the effects of motion blur on the accompanying retinal image. Images were taken with a cooled chargecoupled-device camera (Roper Scientific, Inc., Trenton, New Jersey). To avoid possible edge artifacts from the deformable mirror, imaging was done over a 6 - $\mathrm{mm}$ pupil. A circular fixation target was used to record the relative retinal location of each image (i.e., nasal, temporal, superior, or inferior as well as eccentricity). Images were averaged using a MATLAB-based image-registration algorithm (MathWorks, Inc., Natick, Massachusetts).

In vivo retinal densitometry was combined with this high-resolution retinal-imaging paradigm to identify $\mathrm{S}$ cones. Details on the procedure to classify individual cones as S, M, or L have been published, ${ }^{18,19}$ and only a brief outline is given here. Individual cones in the retinal mosaic were classified by comparing images taken under a fully bleached state [550-nm light of approximately 25 $\times 10^{6}$ troland-seconds] with images taken after 5 min of dark adaptation. The absorptance of the cones in the patch of retina imaged was calculated as 1 minus the ratio of the dark-adapted image to the corresponding fully bleached image. Since the L and M cones absorb strongly at $550 \mathrm{~nm}$, they appear bright in the absorptance image, whereas the $\mathrm{S}$ cones appear dark owing to their low absorptance at $550 \mathrm{~nm}$.

\section{E. Image Analysis}

A freely available image-processing program (ImageJ, National Institutes of Health, Bethesda, Maryland) was used to manually identify the cones in each subject's retinal image. To estimate cone density, the number of cones was divided by the area of retina sampled. The Cartesian $(x, y)$ coordinates of the cones were stored in a text array and used for additional analyses.

To assess the spatial organization of the cone mosaics, the average and SD of nearest-neighbor distances were examined, as well as the Voronoi domains associated with the cones in each mosaic. Voronoi domains were constructed for each cell by defining points in the plane that were closer to that cell than to any other cell in the mosaic. Both metrics are widely used to assess the regularity of cellular mosaics in the retina; see Refs. 20-23. 


\section{RESULTS}

A. Color-Vision Testing and Ophthalmic Examination The father's behavior on all color-vision tests was that of a tritanope. He made tritan errors on the HRR pseudoisochromatic plates, the Lanthony's desaturated D15, and the Farnsworth D15, and his error score on the FM 100Hue was 206 with midpoint at cap 46 [Fig. 1(a)] [normal error score for an individual aged $50-59 \mathrm{yr}$ is $0-130(95 \%$ CI); see Ref. 24], with a significant blue-yellow axis (dif-
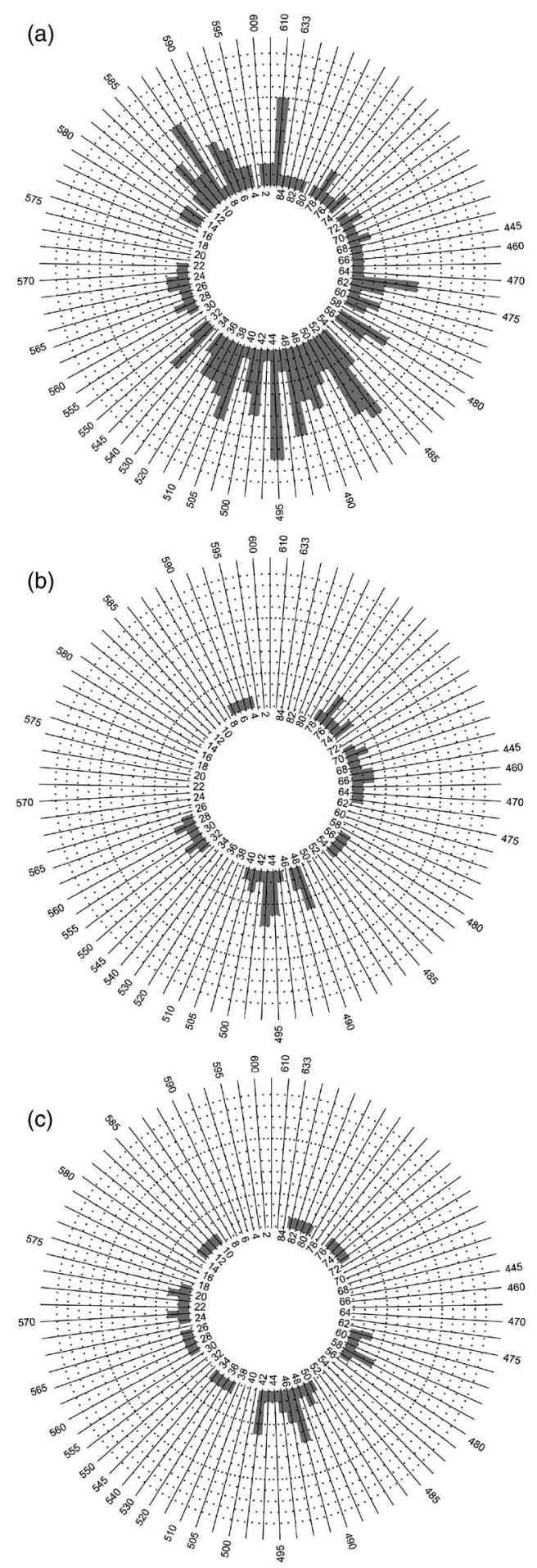

Fig. 1. Results of the Farnsworth-Munsell 100-Hue test for subjects (a) M04 (father), (b) M505 (daughter), (c) M503 (son). ference score between the blue-yellow and the red-green axes was +4.3). ${ }^{25}$ Results from Moreland anomaloscopy and luminance matching showed that he was a tritan with midmatch point (MMP) 14.8 and matching range (MR) 26.9 [normal values (and SD) for the same instrument are MMP 55.1 (5.9), MR 12.0 (1.1)]. ${ }^{26,27}$ His scores on the Cambridge Colour Test (CCT) were 75, 128, and 1100 on the protan (P), deutan (D), and tritan (T) axes of the Trivector test, respectively; the ellipse length was 13.1, and the axis ratio was 551.8 along the tritan axis (angle $99.4 \mathrm{deg}$ ) on field 1 of the Ellipse test ${ }^{14}$ [reported maximum normal values on the Trivector test are 69.3, 82.4, and 113.4 for the P, D, and T axes, respectively, and for field 1 on the Ellipse test are 209.3 for ellipse length and 2.4 for axis ratio ${ }^{28}$ ]. He made no errors on the Ishihara pseudoisochromatic plates, nor on the Medmont C100, and his Rayleigh anomaloscopy and luminance matching were normal with MMP 45.4 and MR 1.9 [normal values (and SD) for the same instrument are MMP 49.6 (3.2), MR 5.5 (1.7)]. ${ }^{26,27}$ He reported that he could not remember having any problems with color vision when he was younger, and it was only in recent years that he has experienced difficulties with discriminating between some colors such as orange-yellow and pink.

The daughter's behavior was as a normal trichromat on the Ishihara pseudoisochromatic plates (no errors), the Farnsworth D15, and Rayleigh and Moreland anomaloscopy and luminance matching (MMP 49.1, MR 1.8; and MMP 50.8, MR 4.2, respectively). She made mild tritan errors on the HRR pseudoisochromatic plates, the Lanthony's desaturated D15, and the FM 100-Hue. Her error score on the FM 100-Hue was 56 with midpoint at cap 44 [Fig. 1(b)] [normal error score for an individual aged $30-39 \mathrm{yr}$ is $0-80(95 \% \mathrm{CI})] ;^{24}$ the difference score between the blue-yellow and the red-green axis was not significant (0.4). ${ }^{25}$ She reported never having experienced problems with discriminating colors.

The son's behavior was also that of a normal trichromat on the Ishihara pseudoisochromatic plates (no errors) and on the Rayleigh and Moreland anomaloscopy and luminance matching (MMP 56.7, MR 1.9; and MMP 56.3, MR 4.2 , respectively). His error score on the FM 100-Hue was 56 with midpoint at cap 46 [Fig. 1(c)]; the difference score between the blue-yellow and the red-green axes was not significant (0.8). ${ }^{25}$

Ophthalmic examinations were normal on both father and daughter, and corrected visual acuities were 6/5 (log MAR-0.1) for both.

\section{B. Genetics}

The father (M04) and both children (M505 and M503) were heterozygous for a nucleotide change that substituted the polar, neutral amino acid glutamine $(\mathrm{Q})$ in place of the positively charged amino acid arginine $(R)$ that is normally present at amino acid position 283 of the S-opsin gene (Fig. 2; see Ref. 29). All 29 control subjects were homozygous for arginine.

\section{Imaging}

Cone density for M505 at 1.25-deg temporal from fixation was 48,916 cones $/ \mathrm{mm}^{2}$, and cone density for M04 was 59,747 cones $/ \mathrm{mm}^{2}$ at 1.0-deg temporal, 


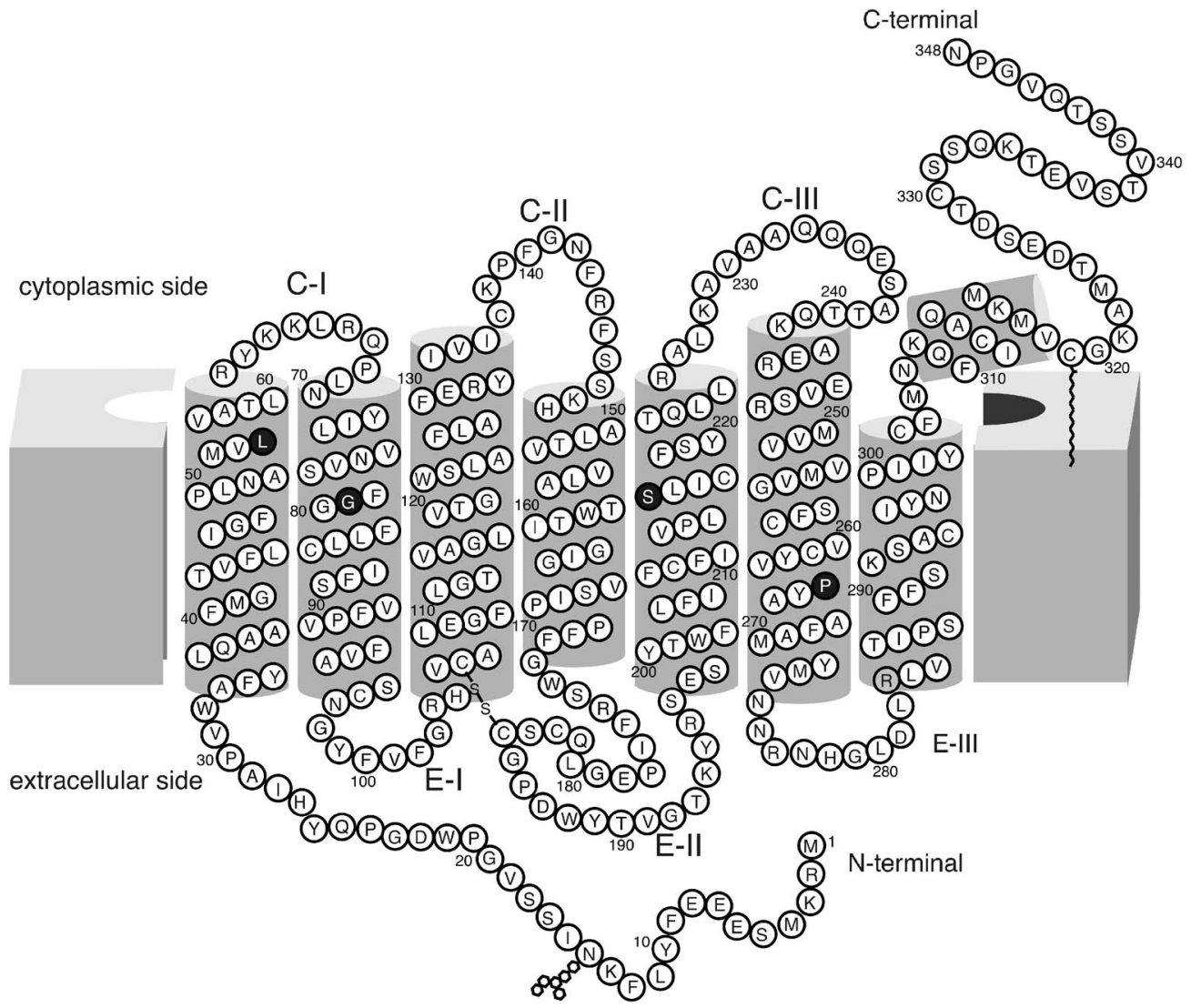

Fig. 2. Model of the S-opsin gene. Circles represent amino acids. The light gray circle indicates the location of the R283Q substitution in the family in this study, who had differing degrees of tritan color-vision deficiency. The other four reported mutations causing tritan color-vision deficiency are indicated by black filled circles: G79R, S214P, P264S, L56P. The figure is modified from Ref. 29 with permission.

Table 1. Statistics of the Spatial Arrangement of the Cone Mosaic

\begin{tabular}{|c|c|c|c|c|c|c|c|c|}
\hline & $\begin{array}{c}\text { Cone } \\
\text { Density }^{a}\end{array}$ & $\begin{array}{c}\text { Mean } \\
\text { Voronoi } \\
\text { Area }^{b}\end{array}$ & $\begin{array}{c}\text { SD } \\
\text { Voronoi } \\
\text { Area }^{b}\end{array}$ & $\begin{array}{c}\text { Mean \# } \\
\text { Voronoi } \\
\text { Sides }\end{array}$ & $\begin{array}{c}\text { SD \# } \\
\text { Voronoi } \\
\text { Sides }\end{array}$ & $\begin{array}{l}\text { Observed } \\
\text { Mean } \\
\text { NND }\end{array}$ & $\begin{array}{c}\text { Expected } \\
\text { Mean } \\
\text { NND }^{c}\end{array}$ & Ratio $^{d}$ \\
\hline \multicolumn{9}{|l|}{$1.0 \mathrm{deg}$} \\
\hline R039 & 44,122 & 23.216 & 3.583 & 5.983 & 0.663 & 4.172 & 4.430 & 0.942 \\
\hline R027 & 66,849 & 14.852 & 2.107 & 5.966 & 0.510 & 3.414 & 3.616 & 0.944 \\
\hline R010 & 75,994 & 12.953 & 1.685 & 5.952 & 0.581 & 3.181 & 3.376 & 0.942 \\
\hline R008 & 53,178 & 18.967 & 2.487 & 5.986 & 0.504 & 3.836 & 4.036 & 0.950 \\
\hline M04 & 59,747 & 16.969 & 2.725 & 5.962 & 0.763 & 3.452 & 3.807 & 0.907 \\
\hline \multicolumn{9}{|l|}{$1.12 \mathrm{deg}$} \\
\hline M04 & 55,575 & 18.266 & 3.200 & 5.964 & 0.737 & 3.578 & 3.948 & 0.906 \\
\hline \multicolumn{9}{|l|}{$1.25 \mathrm{deg}$} \\
\hline R044 & 51,669 & 19.847 & 2.306 & 5.994 & 0.580 & 4.005 & 4.094 & 0.978 \\
\hline M505 & 48,916 & 21.235 & 2.894 & 5.970 & 0.607 & 4.026 & 4.208 & 0.957 \\
\hline R039 & 36,399 & 28.872 & 3.933 & 5.997 & 0.683 & 4.585 & 4.878 & 0.940 \\
\hline R031 & 61,612 & 16.862 & 2.270 & 5.978 & 0.557 & 3.639 & 3.768 & 0.966 \\
\hline R027 & 43,679 & 23.235 & 2.993 & 5.978 & 0.648 & 4.200 & 4.453 & 0.943 \\
\hline M04 & 45,010 & 23.24 & 3.796 & 5.964 & 0.810 & 3.980 & 4.386 & 0.907 \\
\hline
\end{tabular}

${ }^{a}$ Density in cones $/ \mathrm{mm}^{2}$

${ }^{b}$ Area of Voronoi polygons in $\mu \mathrm{m}^{2}$.

${ }^{c}$ Computed assuming a perfectly triangular lattice of cones with a density equal to that observed for a given subject.

${ }^{d}$ Quotient of observed mean NND (nearest-neighbor distance) by expected mean NND; lower values indicate a larger departure from a perfectly triangular arrangement of the cone mosaic. 

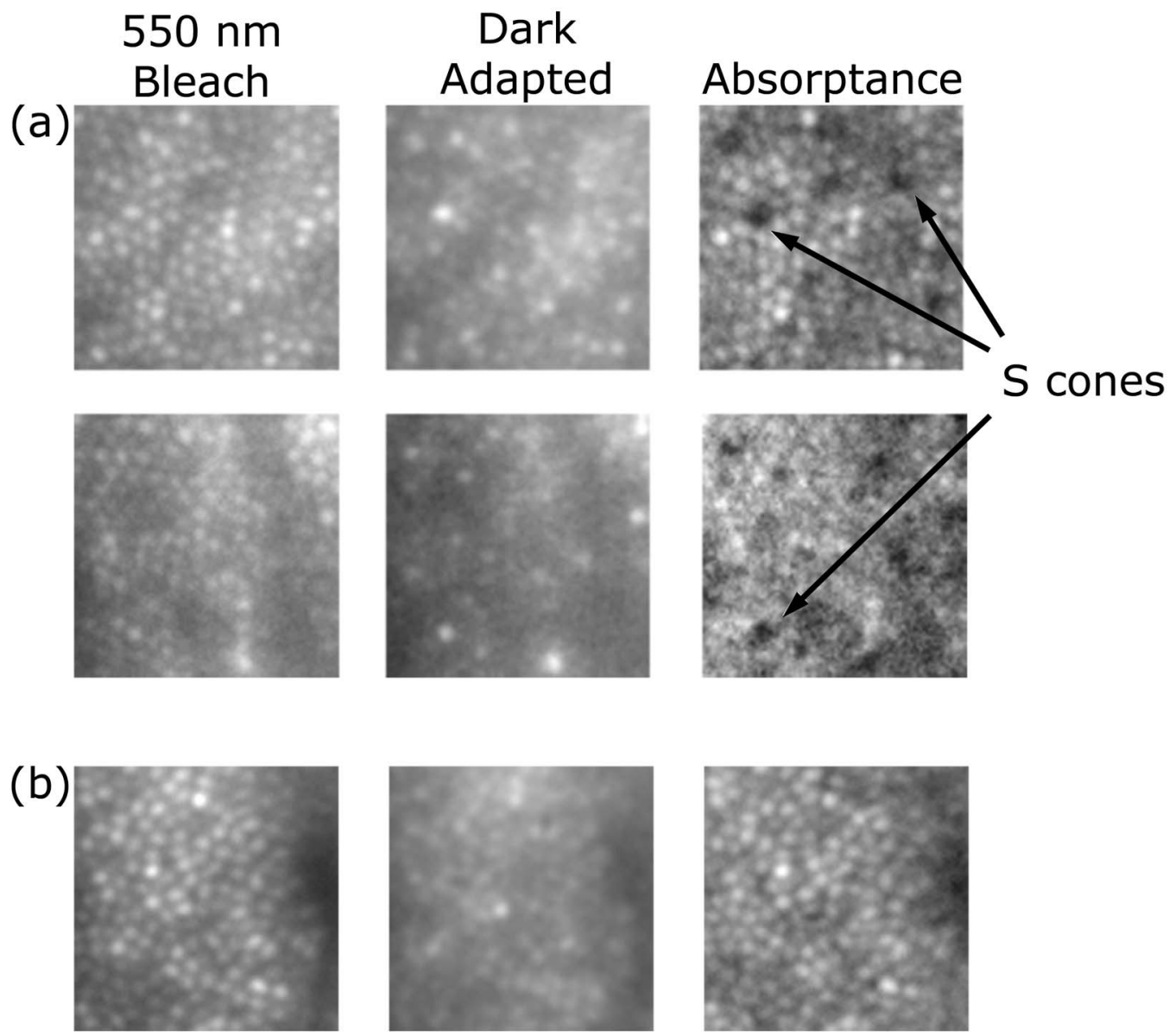

Fig. 3. Retinal images of two tritan subjects. (a) Images from the right eye of the daughter (M505). Numerous cones that are either S cones or empty cones are visible as dark regions in the absorptance image. Given M505's behavioral measurements, and the fact that the number of candidate $\mathrm{S}$ cones is consistent with normal S-cone density, it is probable that they are $\mathrm{S}$ cones. The analysis was done at two different retinal locations at 1.25-deg eccentricity. (b) Images from left eye of the father (M04) at 1.25-deg eccentricity. There is no evidence for S cones. There appears to be some increased irregularity in the mosaic, which may be a signature of cone death.

55,575 cones $/ \mathrm{mm}^{2}$ at 1.12-deg temporal, and 45,010 cones $/ \mathrm{mm}^{2}$ at 1.25 -deg temporal (Table 1 ). Normal values from adaptive optics images are 54,859 (SD 8205) cones $/ \mathrm{mm}^{2}$ at $1.0 \mathrm{deg}(n=14)$ and 44,330 (SD 4724) cones $/ \mathrm{mm}^{2}$ at $1.25 \mathrm{deg}(n=10)$ (data from normal controls combined with data from Refs. 30 and 31).

Figure 3 shows the full bleached, dark-adapted, and absorptance images for M505 (a) and M04 (b). No evidence for $\mathrm{S}$ cones was observed in M04 [Fig. 3(b)]. In M505 about $4.9 \%$ of the cones at $1.25 \mathrm{deg}$ were low absorbing [Fig. 3(a)]. Since S cones absorb negligibly at the imaging and bleaching wavelength of $550 \mathrm{~nm}$, these cones were deemed S cones. Normalized to M505's overall cone density, the density of $\mathrm{S}$ cones at this location was $2224 \mathrm{~S}$ cones $/ \mathrm{mm}^{2}$. In comparison, the average S-cone density in normal trichromatic subjects when measured with adaptive optics at 1.25-deg eccentricity is 2863 (SD 1033) S cones $/ \mathrm{mm}^{2} .^{30}$

\section{Arrangement of the Cone Mosaic}

Figure 4 shows the results of the Voronoi analysis for the tritanope (M04) and two normal trichromats. Figures 4(a)-4(c) illustrate how the Voronoi polygons are derived from the image of the cone mosaic. The control subjects [Figs. 4(d) and 4(e)] were chosen because their cone densities were the closest to M04's cone density at 1.0-deg eccentricity. Seventy percent of cones in the normal mosaics had six neighbors, indicating a regularly packed, triangular mosaic. In contrast, only 55\% of the cones in M04's mosaic had six neighbors [Fig. 4(f)]. Qualitatively, it appears that there are more disruptions in M04's mosaic. In fact, the mean SD in the number of sides on the Voronoi polygons for the control subjects was 0.593 with a SD of 0.064, whereas M04's SD in the number of sides on the Voronoi polygons for his mosaics was $0.763,0.737$, and 0.810 at the three locations tested (Table 1 ). These values are 2.5 SDs from the mean, indicating that his mosaic was significantly more variable than normal $(p=0.0067)$. To further quantify the features of M04's cone mosaic, the mean area of the Voronoi domains in each mosaic was computed (Table 1). As expected, there is a close relationship between the density of cones in the mosaic and the size of the corresponding Voronoi domains, with more dense mosaics having, on average, smaller Voronoi domains. The mosaic variability (expressed as the SD among the Voronoi areas) is also related to the cone density, with more dense mosaics having less variability 

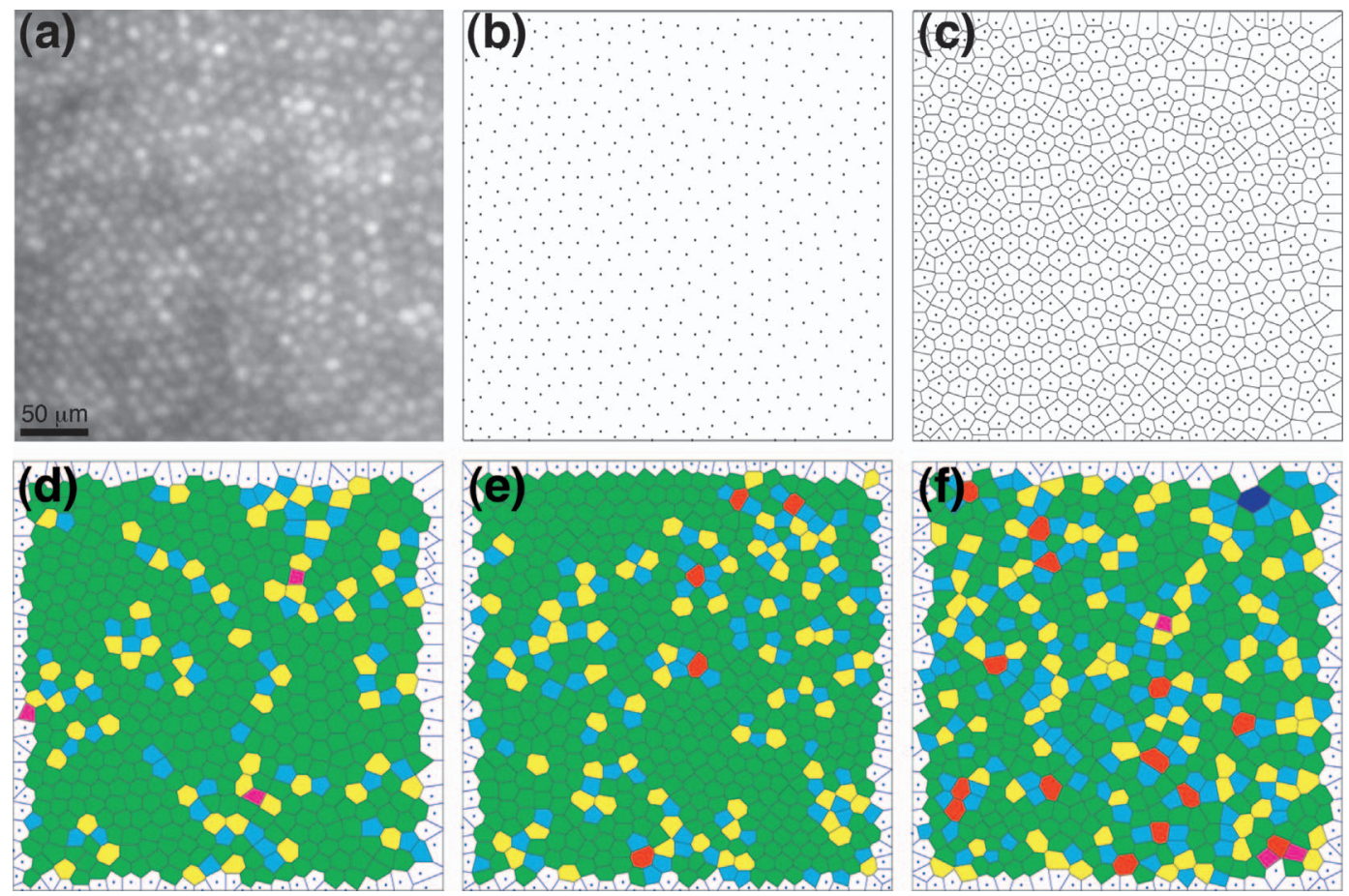

Fig. 4. Analysis of mosaic regularity. (a) Retinal image from a normal trichromat (R008, 1.0 deg). (b) Two-dimensional plot of cone locations from (a). (c) Voronoi domain associated with each cone photoreceptor in (a). (d) Color-coded version of (c), where the color indicates the number of sides on each Voronoi polygon (magenta $=4$, blue $=5$, green $=6$, yellow $=7$, red $=8$, purple $=9$ ). Large regions of sixsided polygons indicate a regular triangular lattice, whereas other colors mark points of disruption in the hexagonal packing of the foveal mosaic. (e) Color-coded Voronoi diagram from a normal trichromat (R031, $1.25 \mathrm{deg}$ ). (f) Color-coded Voronoi diagram from a tritanopic subject (M04, $1.0 \mathrm{deg}$ ). The normal subjects were chosen for comparison because they had cone densities close to that of M04.

[Fig. 5(a)]. Just as there was more variability in the number of sides to the Voronoi polygons in his mosaic, the variability in the area of the Voronoi domains in his mosaic was higher than expected, given his cone density. As seen in Fig. 5(a), both of his data points lie outside the 95\% confidence interval derived from the normal controls.

Also highly correlated with cone density is the mean nearest-neighbor distance (NND). As shown in Fig. 5(b), more dense mosaics have cones that are on average more closely spaced. M04's mean NND (Table 1) is significantly lower than what would be expected based on his cone density. Another way to look at the NND data is to compare the observed mean NND for each subject with that expected from a perfectly triangular mosaic of density identical to that observed for this subject. These values are given in Table 1 and were derived from equations published by Coletta and Williams. ${ }^{32,33}$ By dividing the observed mean NND by the expected mean NND, it is revealed that all control subjects were about $5 \%$ below what was expected. This is probably due to the fact that in the computation of the expected mean NND, uniform density was assumed, although in reality, cone density varies over the area of retina analyzed. Small asymmetries derived from intersubject differences in preferred retinal location for fixation ${ }^{34}$ will affect this ratio as well. Nevertheless, M04's mosaic was significantly different from the controls, as his mean NND was only $90 \%$ of that expected based on his density.

Besides indicating a departure from regularity, the ratio of observed NND to expected NND can be used to infer something about the degree of cone loss in the mosaic. A new NND can be calculated for M04 from the expected mean NND for a mosaic of density identical to that for M04, incorporating the $\sim 95 \%$ reduction in mean NND observed in the controls. New NND values for M04 would then be 3.62 and 3.75 for his 1.0- and 1.25-deg mosaics, respectively. From the relationship between observed mean NND and cone density [Fig. 5(b)], his extrapolated cone density should then have been 61,510 and 57,670 cones $/ \mathrm{mm}^{2}$ for his 1.0 - and 1.25 -deg mosaics, respectively. This is 1763 and 2095 cones $/ \mathrm{mm}^{2}$ below his observed cone density, respectively and this establishes an estimate that he has lost about 1929 cones $/ \mathrm{mm}^{2}$, which is consistent with the mean S-cone density in normal trichromats of 2723 (SD 734) cones $/ \mathrm{mm}^{2}, n=9$ (from Ref. 30) for combined data from 1.0- and 1.25-deg eccentricity, respectively.

It should be noted that in both graphs in Fig. 5, M04's daughter (M505) was included with the normal controls in order to derive the associated regression lines. This was done because normal numbers of $\mathrm{S}$ cones were observed in her mosaic and there were no visible disruptions in the regularity of her mosaic. The inclusion of her data with the normal controls is supported by the fact that her data points lie exactly on the regression line in both graphs.

\section{DISCUSSION}

A major step in understanding the mechanism of inherited tritan color-vision deficiencies was afforded by the landmark work of Weitz and colleagues in identifying 

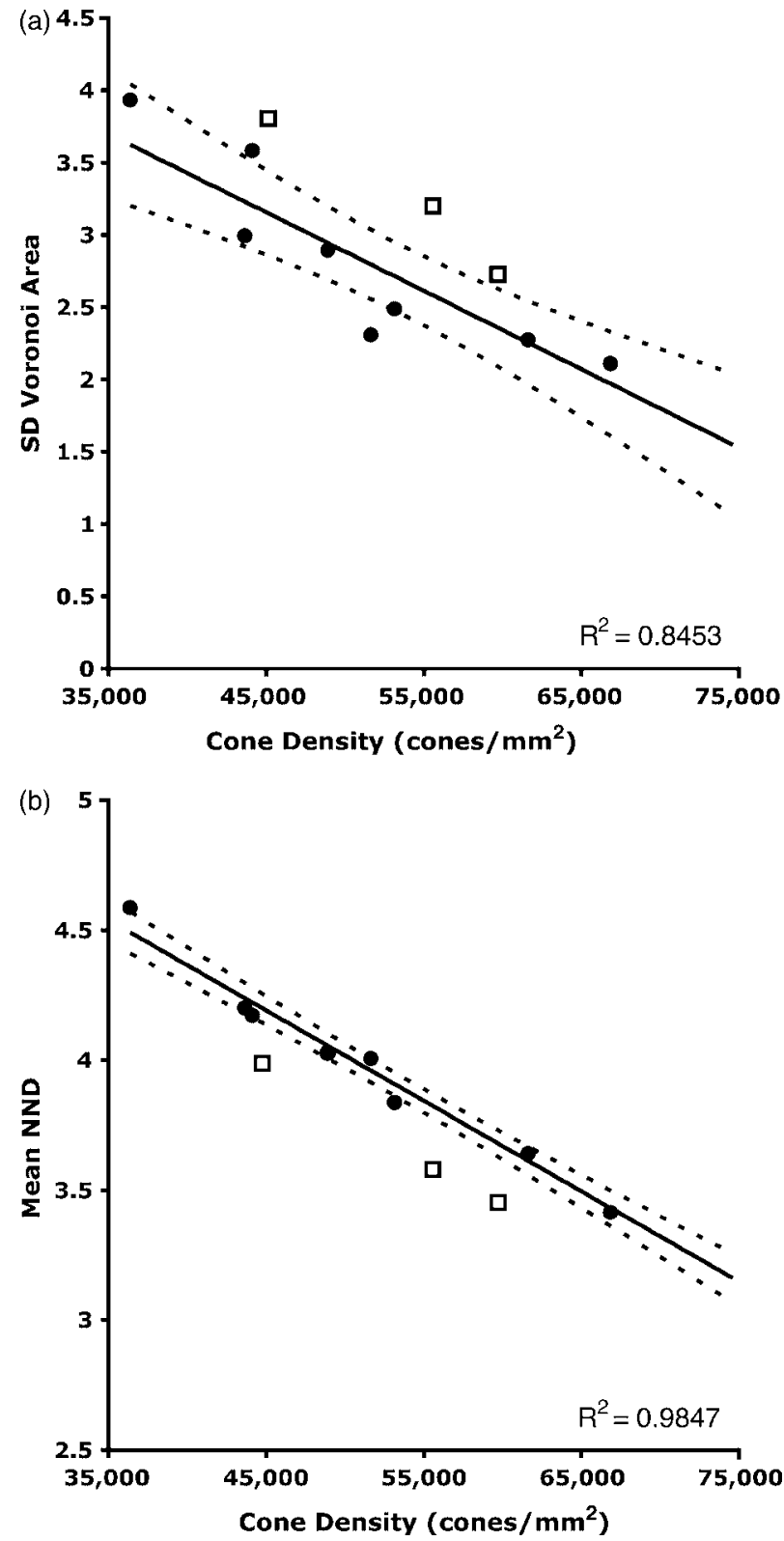

Fig. 5. Correlation of cone density with (a) SD of Voronoi area and (b) mean NND. Filled circles are from normal controls, open squares are from subject M04 (1.0 and $1.25 \mathrm{deg})$. Solid line is best-fitting linear regression, and dashed lines are 95\% confidence intervals.

S-opsin gene mutations in affected individuals. ${ }^{10,11}$ The work reported here provides the first anatomical evidence that tritan phenotypes associated with S-opsin mutations are associated with the loss of $\mathrm{S}$ cones. This suggests a mechanism in which the mutations produce their effects by reducing the viability of the $\mathrm{S}$ cones and that heterozygotes who express both the normal and mutant $\mathrm{S}$ opsins will exhibit trichromatic color vision that can be indistinguishable from normal until the $\mathrm{S}$ cones succumb to the toxicity of the mutant opsin.

It is evident from this study of an affected father and daughter that there is a correlation between behavioral loss of S-cone function and physical disruption in the cone mosaic. $\mathrm{S}$ cones are sparse in the normal retina, comprising about $5 \%$ of the photoreceptors, with their highest density $\left(>2000\right.$ cones $\left./ \mathrm{mm}^{2}\right)$ at about 10-deg eccentricity, ${ }^{35}$ but with large variations between individuals. ${ }^{36}$ The difference between father and daughter in this study, however, is too large to be considered as within-normal intersubject variability: The daughter showed near-normal S-cone density and the father a complete loss at the eccentricity where peak S-cone density normally occurs. ${ }^{34}$ The expectation is that the number of $\mathrm{S}$ cones in the daughter's mosaic would be further reduced if she were imaged later in life. The fact that the father's total cone density was still within the normal range even if he had lost all his $\mathrm{S}$ cones is not surprising, as the normal variation in total cone density exceeds $5 \%$. $^{18,36,37}$

One limitation of the present study is the absence of age-matched controls for the analysis of the spatial topography of the tritanope mosaic of the father. It might be argued that, as part of the normal aging process, a significant number of foveal cones are lost, thereby disrupting mosaic regularity. In fact, while maximum cone density varies between individuals, the number of foveal cones remains relatively constant throughout adulthood in the absence of retinal disease. ${ }^{38,39}$ Since disruption of the mosaic is unlikely to be part of the normal aging process, it follows that it is the loss of $\mathrm{S}$ cones that is responsible for mosaic irregularity in the tritanope. In support of this conclusion, Hoang et al. ${ }^{40}$ detected no disturbance of the mosaic in their analysis of histological data from a 68 -year-old retina at the same retinal location as that studied here (data not shown).

The Voronoi and nearest-neighbor analyses suggest that some reorganization of the cone mosaic took place after the $\mathrm{S}$ cones had died: The $\mathrm{L}$ and $\mathrm{M}$ cones seem to have at least partially filled in the gaps that previously were taken up by $\mathrm{S}$ cones, making the father's mosaic look irregular in comparison with the triangular packing seen in that of the daughter and normal trichromatic subjects $^{18,19,35}$; see also Fig. 4. This irregularity suggests that the $\mathrm{S}$ cones had completely degenerated after foveal migration was complete. ${ }^{41-43}$ Mosaic regularity is preserved in early cone loss where foveal migration has acted to repack the cones ${ }^{31}$ or in other cases where normal foveal migration has occurred but a functional opsin is not expressed in the cones. ${ }^{44}$ The disruption in mosaic regularity, together with the absence of $\mathrm{S}$ cones, is the only visible change in the retina. Thus, despite normal cone density, there are other signatures in the retinal images that can be used. In fact, given how variable cone density is among normal controls, measures of regularity may be more sensitive to the very early stages of cone loss in other photoreceptor degenerations.

Until now, the effects of amino acid substitutions on $\mathrm{S}$ cones were unknown, but the absence of $\mathrm{S}$ cones coupled with the decreased density and abnormal packing arrangement of the cones in a tritan subject (M04) suggests that heterozygosity for the R283Q mutation ultimately results in the death of $\mathrm{S}$ cones. S-opsin mutations that cause autosomal dominant tritan color-vision deficiencies are analogous to rhodopsin mutations that cause autosomal dominant retinitis pigmentosa, in which dominant negative interactions between normal and mutant pigments expressed in the same photoreceptor lead to the 
death of the affected photoreceptor. ${ }^{45,46}$ The molecular genetic and imaging results reported here along with previous molecular genetic data support the hypothesis that the phenotypic difference between the father and the daughter with the same mutation can be attributed to their being at different stages in the progression in which dominant negative interactions have compromised the function and viability of S cones. The observation of Went and Pronk ${ }^{8}$ that error scores on color-vision tests tend to be higher with older tritan subjects than with younger ones also supports this hypothesis. Further, the imaging results are consistent with the loss of S-cone function being due directly to the physical loss of $\mathrm{S}$ cones as a result of the mutant S-opsin pathology rather than to the normal aging process. ${ }^{47}$

Some studies ${ }^{4,48}$ have attempted to classify tritan deficiencies into dichromatic and anomalous trichromatic forms by analogy with red-green color-vision deficiencies, and a third form, incomplete tritanopia, has also been proposed, ${ }^{3,6}$ but the phenotype is not clearly different from tritanomaly. ${ }^{3}$ A rationale for a tritan phenotype that is analogous to red-green anomalous trichromacy is difficult to justify. Recall that the red-green anomalous trichromacies are caused by the combination of the loss of one class of photoreceptor, L or M, and the addition of a second subtype of the remaining class such that color vision in the red-green region of the spectrum is mediated by two cone types whose spectral sensitivities overlap much more than in normal trichromacy. ${ }^{49}$ In contrast to the 20-30 nm that separate L from M pigments in normal color vision, ${ }^{50,51}$ the spectral separation between the L-cone subtypes or M-cone subtypes in anomalous trichromacy ranges from about 1 to about $12 \mathrm{~nm}$ and, as expected, the degree to which color discrimination is impaired is directly related to the magnitude of the spectral separation. ${ }^{14,52,53}$ Spectral-tuning studies of mammalian short-wavelength-sensitive pigments that are evolutionarily closely related to the human S-cone photopigment have identified amino acid substitutions that are capable of shifting the spectrum over the range from about 359 to $423 \mathrm{~nm} .{ }^{54,55}$ But the human S-cone photopigment peaks at $415-419 \mathrm{~nm},{ }^{56,57}$ and no amino acid substitutions that affect the S-cone spectrum have been identified in human subjects. Indeed, a shift of about $100 \mathrm{~nm}$ in S-cone sensitivity to long wavelengths would be required to produce a degree of overlap between the $\mathrm{S}$ - and the M-cone sensitivity that is equivalent to the overlap between M- or L-cone subtypes in red-green deficiencies. ${ }^{52,53}$ Such a large shift would not be accomplished with a single amino acid substitution, ${ }^{55}$ and the anomalous trichromatic tritan phenotype has instead been attributed to the involvement of rods in the color match made by tritan subjects. ${ }^{7}$

Historically, the existence of congenital tritan colorvision deficiencies has been controversial. ${ }^{58,59}$ The incomplete penetrance and general trend of worsening colorvision test scores with increasing age among tritan subjects $^{8}$ suggests that, at least in some affected individuals, inherited tritan color-vision deficiency is not congenital (i.e., present at birth). The color-vision deficiency associated with mutation in the S-cone opsins is expected to be present from birth in individuals homozygous for a mu- tation, but not necessarily for heterozygous individuals. This is because the potential for dominant negative interactions between normal and mutant pigments is absent in homozygotes. By the same reasoning, homozygous S-opsin mutations would not necessarily be expected to produce a progressive degeneration, but instead a stationary disorder of the $\mathrm{S}$ cones. Although homozygous individuals are thought to be exceptionally rare, tritan individuals from two of the five pedigrees that have been studied in detail contain individuals who are homozygous for an S-opsin mutation, but there are also individuals within these two pedigrees who are heterozygous. ${ }^{10}$ Within the same pedigree, it would be expected that a younger subject who is homozygous would perform more poorly than an older subject who is heterozygous. Recruitment tactics for identifying tritan subjects for experimental analysis tend to select for rare events such as homozygotes. Most individuals with inherited tritan deficiencies are heterozygous and, according to the hypothesis supported by the experimental data presented here, they need not exhibit complete dichromacy until the disorder has progressed to a stage when a critical number of $\mathrm{S}$ cones have lost viability. Variability in phenotype probably derives primarily from differences in the degree to which S-cone function is diminished.

\section{ACKNOWLEDGMENTS}

This work was supported by the Wellcome Trust (grant 064669/Z/01/Z), NIH [grants EY04367, EY09303, EY09620, and F32EY014789 (K. L. Gunther)], Research to Prevent Blindness, and the Norwegian Research Council (grant 176623/V00). We thank K. Amano, L. Chen, R. F. Itzhaki, J. Neitz, J. Wolfing, and M. A. Wozniak for their assistance, and K. Palczewski for providing us with material for Fig. 2.

E-mail correspondence may be addressed to R. C. Baraas at rigmor.baraas@hibu.no.

\section{REFERENCES}

1. W. D. Wright, "The characteristics of tritanopia," J. Opt. Soc. Am. 42, 509-521 (1952).

2. T. Piantanida, "Genetics of inherited colour vision deficiencies," in Inherited and Acquired Colour Vision Deficiencies: Fundamental Aspects and Clinical Studies, D. H. Foster, ed. (Macmillan, 1991), pp. 88-114.

3. B. L. Cole, G. H. Henry, and J. Nathan, "Phenotypical variations of tritanopia," Vision Res. 6, 301-313 (1966)

4. H. Kalmus, "The familial distribution of congenital tritanopia, with some remarks on some similar conditions," Ann. Hum. Genet. 20, 39-56 (1955).

5. Y. Miyake, K. Yagasaki, and H. Ichikawa, "Differential diagnosis of congenital tritanopia and dominantly inherited juvenile optic atrophy," Arch. Ophthalmol. (Chicago) 103, 1496-1501 (1985).

6. T. Neuhann, H. Kalmus, and W. Jaeger, "Ophthalmological findings in the tritans, described by Wright and Kalmus," Mod. Probl. Ophthalmol. 17, 135-142 (1976).

7. J. Pokorny, V. C. Smith, and L. N. Went, "Color matching in autosomal dominant tritan defect," J. Opt. Soc. Am. 71, 1327-1334 (1981).

8. L. N. Went and N. Pronk, "The genetics of tritan disturbances," Hum. Genet. 69, 255-262 (1985).

9. K. L. Gunther, J. Neitz, and M. Neitz, "A novel mutation in the short-wavelength-sensitive cone pigment gene 
associated with a tritan color vision defect," Visual Neurosci. 23, 403-409 (2006).

10. C. J. Weitz, Y. Miyake, K. Shinzato, E. Montag, E. Zrenner, L. N. Went, and J. Nathans, "Human tritanopia associated with two amino acid substitutions in the blue-sensitive opsin," Am. J. Hum. Genet. 50, 498-507 (1992).

11. C. J. Weitz, L. N. Went, and J. Nathans, "Human tritanopia associated with a third amino acid substitution in the blue-sensitive visual pigment," Am. J. Hum. Genet. 51, 444-446 (1992).

12. J. Hwa, P. Garriga, X. Liu, and H. G. Khorana, "Structure and function in rhodopsin: packing of the helices in the transmembrane domain and folding to a tertiary structure in the intradiscal domain are coupled," Proc. Natl. Acad. Sci. U.S.A. 94, 10571-10576 (1997).

13. V. C. Sheffield, G. A. Fishman, J. S. Beck, A. E. Kimura, and E. M. Stone, "Identification of novel rhodopsin mutations associated with retinitis pigmentosa by GCclamped denaturing gradient gel electrophoresis," Am. J. Hum. Genet. 49, 699-706 (1991).

14. B. C. Regan, J. P. Reffin, and J. D. Mollon, "Luminance noise and the rapid determination of discrimination ellipses in colour deficiency," Vision Res. 34, 1279-1299 (1994)

15. H. Hofer, P. Artal, B. Singer, J. L. Aragón, and D. R. Williams, "Dynamics of the eye's wave aberration," J. Opt. Soc. Am. A 18, 497-506 (2001).

16. J. Liang, D. R. Williams, and D. T. Miller, "Supernormal vision and high-resolution retinal imaging through adaptive optics," J. Opt. Soc. Am. A 14, 2884-2892 (1997).

17. A. Pallikaris, D. R. Williams, and H. Hofer, "The reflectance of single cones in the living human eye," Invest. Ophthalmol. Visual Sci. 44, 4580-4592 (2003).

18. A. Roorda, A. B. Metha, P. Lennie, and D. R. Williams, "Packing arrangement of the three cone classes in primate retina," Vision Res. 41, 1291-1306 (2001).

19. A. Roorda and D. R. Williams, "The arrangement of the three cone classes in the living human eye," Nature $\mathbf{3 9 7}$, 520-522 (1999).

20. L. Galli-Resta, E. Novelli, Z. Kryger, G. H. Jacobs, and B. E. Reese, "Modelling the mosaic organization of rod and cone photoreceptors with a minimal-spacing rule," Eur. J. Neurosci. 11, 1461-1469 (1999)

21. M. B. Shapiro, S. J. Schein, and F. M. de Monasterio, "Regularity and structure of the spatial pattern of blue cones of the Macaque retina," J. Am. Stat. Assoc. 803-812 (1985)

22. H. Wässle and H. J. Riemann, "The mosaic of nerve cells in the mammalian retina," Proc. R. Soc. London, Ser. B 200, 441-461 (1978).

23. X. J. Zhan and J. B. Troy, "Modeling cat retinal beta-cell arrays," Visual Neurosci. 17, 23-39 (2000).

24. P. R. Kinnear and A. Sahraie, "New Farnsworth-Munsell 100 hue test norms of normal observers for each year of age 5-22 and for age decades 30-70," Br. J. Ophthamol. 86, 1408-1411 (2002).

25. V. C. Smith, J. Pokorny, and A. S. Pass, "Color-axis determination on the Farnsworth-Munsell 100-hue test," Am. J. Ophthalmol. 100, 176-182 (1985).

26. A. Kurtenbach, U. Schiefer, A. Neu, and E. Zrenner, "Preretinopic changes in the colour vision of juvenile diabetics," Br. J. Ophthamol. 83, 43-46 (1999).

27. M. Pelizzone, J. Sommerhalder, A. Roth, and D. Hermès, "Automated Rayleigh and Moreland matches on a computer-controlled anomaloscope," in Colour Vision Deficiencies, B. Drum, J. D. Moreland, and A. Serra, eds. (Kluwer, 1991), pp. 151-159.

28. D. F. Ventura, L. C. L. Silveira, A. R. Rodrigues, J. M. De Souza, M. Gualtieri, D. Bonci, and M. F. Costa, "Preliminary norms for the Cambridge Colour Test," in Normal \& Defective Colour Vision, J. D. Mollon, J. Pokorny, and K. Knoblauch, eds. (Oxford U. Press, 2003), pp. 331-339.

29. R. E. Stenkamp, S. Filipek, C. A. G. G. Driessen, D. C. Teller, and K. Palczewski, "Crystal structure of rhodopsin: a template for cone visual pigments and other G protein- coupled receptors," Biochim. Biophys. Acta 1565, 168-182 (2002).

30. H. Hofer, J. Carroll, J. Neitz, M. Neitz, and D. R. Williams, "Organization of the human trichromatic cone mosaic," J. Neurosci. 25, 9669-9679 (2005).

31. J. Carroll, M. Neitz, J. Wolfing, D. Gray, J. Neitz, and D. R. Williams, "Different genetic causes of red-green color blindness give rise to different retinal phenotypes as assessed with adaptive optics," Invest. Ophthalmol. Visual Sci. 45, E-Abstract 4341 (2004).

32. N. J. Coletta and D. R. Williams, "Psychophysical estimate of extrafoveal cone spacing," J. Opt. Soc. Am. A 4, 1503-1513 (1987).

33. D. R. Williams and N. J. Coletta, "Cone spacing and the visual resolution limit,” J. Opt. Soc. Am. A 4, 1514-1523 (1987).

34. N. M. Putnam, H. J. Hofer, N. Doble, L. Chen, J. Carroll, and D. R. Williams, "The locus of fixation and the foveal cone mosaic," J. Vision 5, 632-639 (2005).

35. C. A. Curcio, K. A. Allen, K. R. Sloan, C. L. Lerea, J. B. Hurley, I. B. Klock, and A. H. Milam, "Distribution and morphology of human cone photoreceptors stained with anti-blue opsin,” J. Comp. Neurol. 312, 610-624 (1991)

36. C. A. Curcio, K. R. Sloan, R. E. Kalina, and A. E. Hendrickson, "Human photoreceptor topography," J. Comp. Neurol. 292, 497-523 (1990).

37. H. Gao and J. G. Hollyfield, "Aging of the human retina. Differential loss of neurons and retinal pigment epithelial cells," Invest. Ophthalmol. Visual Sci. 33, 1-17 (1992).

38. P. D. Spear, "Neural bases of visual deficits during aging," Vision Res. 33, 2589-2609 (1993).

39. G. R. Jackson, C. Owsley, and C. A. Curcio, "Photoreceptor degeneration and dysfunction in aging and age-related maculopathy," Aging Res. Rev. 1, 381-396 (2002).

40. Q. V. Hoang, R. A. Linsenmeier, C. K. Chung, and C. A. Curcio, "Photoreceptor inner segments in monkey and human retina: mitochondrial density, optics, and regional variation," Visual Neurosci. 19, 395-407 (2002).

41. A. E. Hendrickson and C. Yuodelis, "The morphological development of the human fovea," Ophthalmology 91, 603-612 (1984).

42. C. Yuodelis and A. Hendrickson, "A qualitative and quantitative analysis of the human fovea during development," Vision Res. 26, 847-855 (1986).

43. C. Diaz-Araya and J. M. Provis, "Evidence of photoreceptor migration during early foveal development: a quantitative analysis of human fetal retinae," Visual Neurosci. 8, 505-514 (1992).

44. J. Carroll, M. Neitz, H. Hofer, J. Neitz, and D. R. Williams, "Functional photoreceptor loss revealed with adaptive optics: an alternate cause of color blindness," Proc. Natl. Acad. Sci. U.S.A. 101, 8461-8466 (2004).

45. T. P. Dryja, L. B. Hahn, G. S. Cowley, T. L. McGee, and E. L. Berson, "Mutation spectrum of the rhodopsin gene among patients with autosomal dominant retinitis pigmentosa," Proc. Natl. Acad. Sci. U.S.A. 88, 9370-9374 (1991).

46. C. H. Sung, C. M. Davenport, J. C. Hennessey, I. H. Maumenee, S. G. Jacobson, J. R. Heckenlively, R. Nowakowski, G. Fishman, P. Gouras, and J. Nathans, "Rhodopsin mutations in autosomal dominant retinitis pigmentosa," Proc. Natl. Acad. Sci. U.S.A. 88, 6481-6485 (1991).

47. C. A. Curcio, C. L. Millican, K. A. Allen, and R. E. Kalina, "Aging of the human photoreceptor mosaic: evidence for selective vulnerability of rods in central retina," Invest. Ophthalmol. Visual Sci. 34, 3278-3296 (1993).

48. G. H. Henry, B. L. Cole, and J. Nathan, "The inheritance of congenital tritanopia with the report of an extensive pedigree," Ann. Hum. Genet. 27, 219-231 (1964).

49. M. Neitz and J. Neitz, "Molecular genetics of color vision and color vision defects," Arch. Ophthalmol. (Chicago) 118, 691-700 (2000).

50. J. Carroll, J. Neitz, and M. Neitz, "Estimates of L:M cone ratio from ERG flicker photometry and genetics," J. Vision 2, 531-542 (2002). 
51. L. T. Sharpe, A. Stockman, H. Jägle, H. Knau, G. Klausen, A. Reitner, and J. Nathans, "Red, green, and red-green hybrid pigments in the human retina: correlations between deduced protein sequences and psychophysically measured spectral sensitivities," J. Neurosci. 18, 10053-10069 (1998).

52. S. K. Shevell, J. C. He, P. Kainz, J. Neitz, and M. Neitz, "Relating color discrimination to photopigment genes in deutan observers," Vision Res. 38, 3371-3376 (1998).

53. J. Neitz, M. Neitz, and P. M. Kainz, "Visual pigment gene structure and the severity of color vision defects," Science 274, 801-804 (1996).

54. J. W. L. Parry, S. Poopalasundaram, J. K. Bowmaker, and D. M. Hunt, "A novel amino acid substitution is responsible for spectral tuning in a rodent violet-sensitive visual pigment," Biochemistry 43, 8014-8020 (2004).

55. S. Yokoyama, W. T. Starmer, Y. Takahashi, and T. Tada,
"Tertiary structure and spectral tuning of UV and violet pigments in vertebrates," Gene 365, 95-103 (2006).

56. J. I. Fasick, N. Lee, and D. D. Oprian, "Spectral tuning in the human blue cone pigment," Biochemistry 38, 11593-11596 (1999)

57. H. J. Dartnall, J. K. Bowmaker, and J. D. Mollon, "Human visual pigments: microspectrophotometric results from the eyes of seven persons," Proc. R. Soc. London, Ser. B 220, $115-130$ (1983).

58. A. E. Krill, V. C. Smith, and J. Pokorny, "Further studies supporting the identity of congenital tritanopia and hereditary dominant optic atrophy," Invest. Ophthalmol. 10, 457-465 (1971).

59. D. P. Smith, B. L. Cole, and A. Isaacs, "Congenital tritanopia without neuroretinal disease," Invest. Ophthalmol. 12, 608-617 (1973). 